\title{
Direct determination of free exciton binding energy from phonon-assisted luminescence spectra in GaN epilayers
}

\author{
S. J. $\mathrm{Xu}^{\mathrm{a})}$ \\ Department of Physics and HKU-CAS Joint Laboratory on New Materials, the University of Hong Kong, \\ Pokfulam Road, Hong Kong, China \\ W. Liu and M. F. Li \\ Department of Electrical and Computer Engineering, National University of Singapore, Singapore 117576, \\ Singapore
}

(Received 5 June 2002; accepted 24 August 2002)

\begin{abstract}
Investigation of variable-temperature longitudinal optical (LO) phonon-assisted luminescence spectra of free excitons and free electrons in heteroepitaxial $\mathrm{GaN}$ thin films has been conducted. It is found that thermal broadening of the LO phonon-assisted photoluminescence peaks is much slower than those of the peaks of their parents so that the first-order LO peaks of the free exciton transition and the band-to-band transition can be well resolved even at room temperature, leading to a direct determination of the band $A$ free exciton binding energy as $25.4 \pm 0.9 \mathrm{meV}$. At the same time, we demonstrate that the simple hydrogenlike model still is a good approximation to describe the energy level structure of free excitons in GaN. (C) 2002 American Institute of Physics.
\end{abstract}

[DOI: $10.1063 / 1.1514391]$

Wurtzite $\mathrm{GaN}$ is a polar semiconductor with a direct band gap. Currently, it is of great interest for shortwavelength optoelectronic and high-power and hightemperature electronic device applications. It is known that excitonic radiative transitions play a fundamental role in the photoluminescence (PL) processes in high-quality undoped heteroepitaxial GaN films even at room temperature. Among them, the $\Gamma_{9 V}$ band free exciton transition (denoted FXA in the article) is often used as a reference line to mark other exciton transitions. Thus, the binding energy of this exciton is very important for understanding optical properties of GaN. So far, very scattered values from 18 to $28 \mathrm{meV}$ have been reported. ${ }^{1-8}$ This implies that the binding energy of the $A$ free excitons depends most likely on the residual strain in the heteroepitaxial GaN layers. ${ }^{1}$ Most of these experimental values are calculated from the measured energy separation between $1 S$ and $2 S$ states of the $A$ free excitons in the optical spectroscopy using a simple hydrogenlike model. In this letter, we determine the binding energy of the $A$ free excitons directly from the energy distance between longitudinal optical (LO) phonon peaks of the free exciton transition and the band-to-band transition in the GaN epilayer, which is $\sim 25.4 \mathrm{meV}$

The sample used in this work was nominally undoped GaN epilayer grown on sapphire by metalorganic chemical vapor epitaxy (MOVPE). The thickness of the layer is 2.88 $\mu \mathrm{m}$. In the PL measurements, the sample was excited with the $325 \mathrm{~nm}$ line of a $\mathrm{He}-\mathrm{Cd}$ laser with output power of 40 $\mathrm{mW}$. The luminescent signal was dispersed and detected with a SPEX 750M monochromator equipped with a R928 photomultiplier. The temperature of the sample can be tuned from 3.5 to $300 \mathrm{~K}$ with a Janis closed-cycle cryostat.

At low temperatures, the shallow neutral donor-bound

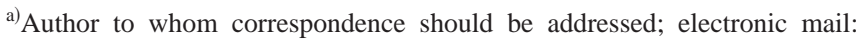
sjxu@hkucc.hku.hk
}

exciton transition $\left(D^{0}, X\right)$ dominates in the PL spectra, as shown in Fig. 1. It was found that the linewidth of the $D^{0}, X$ peak at $3.5 \mathrm{~K}$ was as narrow as $2.4 \mathrm{meV}$ under low power excitation. ${ }^{9}$ This indicates a very high quality of the sample investigated in the present work. Currently, the highest quality heteroepitaxial GaN films grown by MOVPE have a $D^{0}, X$ linewidth between 2 to $3 \mathrm{meV}$ at low temperature. ${ }^{10}$ Besides the dominant $D^{0}, X$ peak, the FXA $(n=1)$, the FXB $(n=1)$, and the FXA $(n=2)$ can be resolved in Fig. 1. Here FXB stands for the $B$ free exciton transition related to $\Gamma_{7 V}$. From Fig. 1, it can be seen that the energy separation between $n=1$ state ( $1 S$ state) and $n=2$ state ( $2 S$ state) of the $A$ free exciton is $\sim 18.7 \mathrm{meV}$. The binding energy of the $A$ free exciton can be calculated from such energy separation assuming the hydrogenic model based on the effective-mass approximation. According to Elliott's theory, ${ }^{11}$ exciton states are given by

$$
E_{n}=-\frac{E_{b}}{n^{2}}
$$

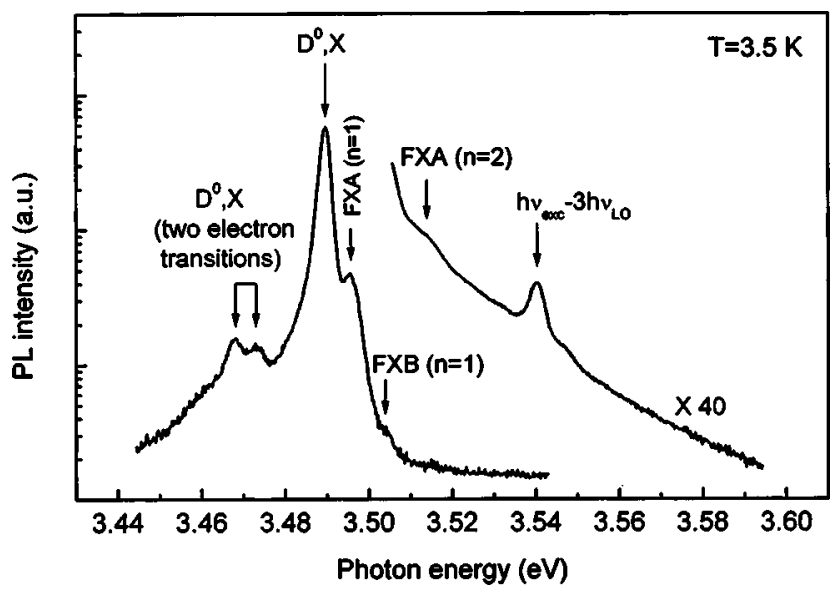

FIG. 1. $3.5 \mathrm{~K}$ exciton resonant PL spectrum of the GaN epilayer. 


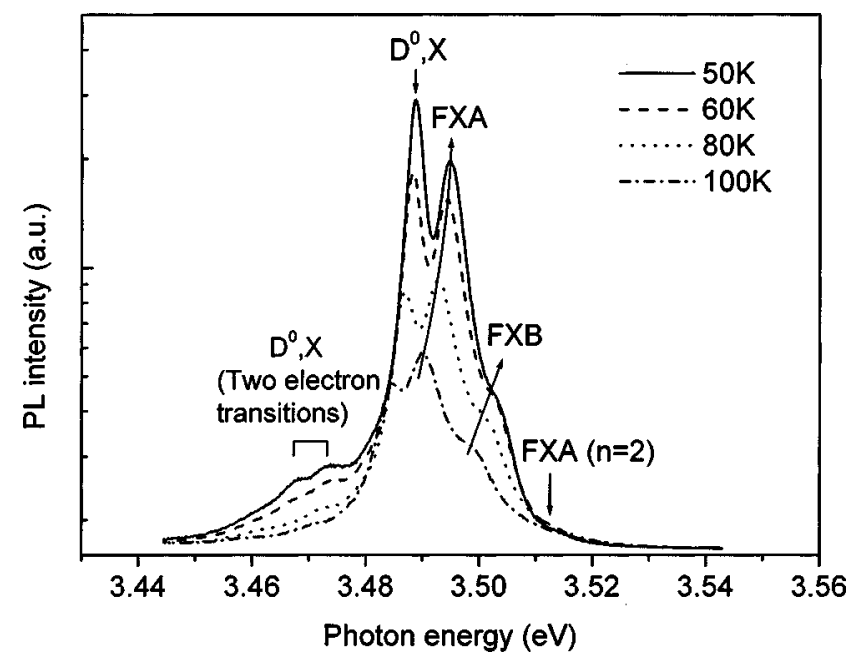

FIG. 2. Measured exciton resonant PL spectra of the GaN epilayer at different temperatures.

where $E_{b}$ is the exciton binding energy and $n$ is an integer. The binding energy of the $A$ free exciton is hence calculated to be $24.9 \mathrm{meV}$.

We also observed a pair of weak peaks referred to as $D^{0}, X$ (two electron transitions), as shown in Fig. 1. They are special and interesting recombination processes. In such a recombination process, the donor electron of a $D^{0}, X$ is transferred from its ground state into an excited state. ${ }^{12}$ The energies of two peaks observed here are $16.4 \mathrm{meV}$ and 21.6 meV lower than that of the $D^{0}, X$ line, respectively. Therefore, our sample appears to contain two residual donors with binding energies of $\sim 22$ and $28.8 \mathrm{meV}$, respectively, assuming Eq. (1) is also applicable to the case of the donor-bound excitons. The $\sim 22 \mathrm{meV}$ donor was recently identified as $\mathrm{Si}^{13}{ }^{13}$ Skromme et al. ${ }^{8}$ observed the $\sim 28.8 \mathrm{meV}$ donor in their MOVPE sample too. However, the origin of this residual donor has not yet identified. The existence of two residual donors in the sample studied here was confirmed by the first-order LO phonon-assisted spectra at low temperature. ${ }^{9}$ In addition, an unknown peak at $3.5402 \mathrm{eV}$ is clearly seen in Fig. 1. From its spectral features, such as high energy and relatively strong oscillator strength, the $C$ free exciton transition should be ruled out as the origin of this line. It should be noted that its energy separation from the excitation laser line $(325 \mathrm{~nm}, 3.8154 \mathrm{eV})$ is just three times that of the LO phonon characteristic energy $(\sim 92 \mathrm{meV}) .{ }^{9}$ We thus believe that it stems from third-order LO phonon Raman scattering.

A pronounced relative-intensity change of the neutral donor-bound exciton and the free exciton transitions is observed as the temperature is elevated. A rapid increase in the relative intensity of FXA with respect to $D^{0}, X$ occurs due to thermalization of the bound excitons into free excitons. At about $70 \mathrm{~K}$, the $D^{0}, X$ line loses its dominant position, as shown in Fig. 2. It is interesting that the LO phonon peak of $D^{0}, X$ quenches more rapidly than itself. Only at about $25 \mathrm{~K}$, the phonon peaks of the $A$ free excitons becomes stronger than those of the neutral donor-bound excitons. ${ }^{9}$ When the temperature is raised to $50 \mathrm{~K}$, its phonon peaks almost disappear while the $D^{0}, X$ transition still dominates. The mechanism behind this phenomenon is not clear and would need

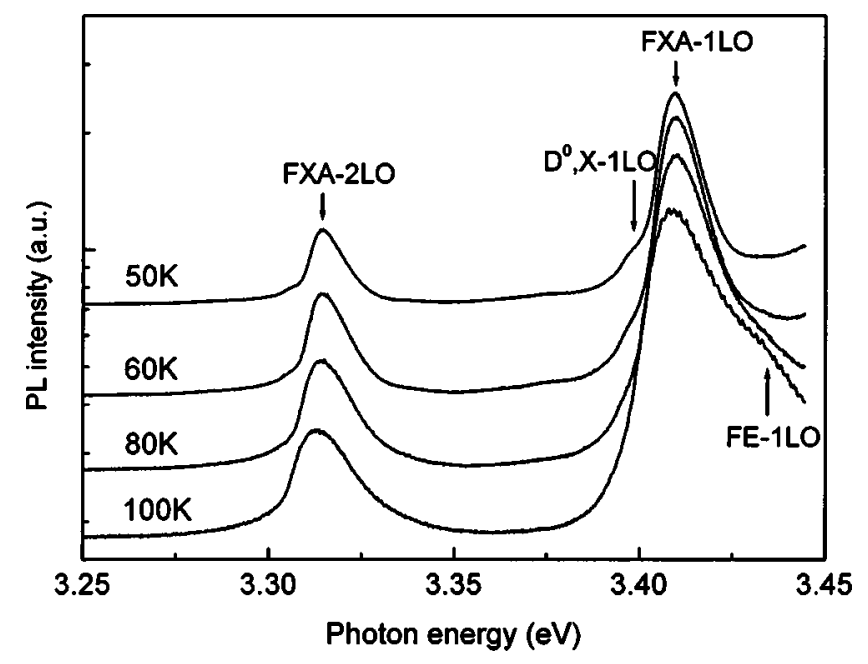

FIG. 3. Corresponding phonon peaks of the principal luminescent lines in Fig. 2.

more investigation. The further spectral evolution of the LO phonon-assisted exciton transitions with temperature is more interesting. Figure 3 shows LO phonon-assisted luminescence spectra measured in temperature range from 50 to 100 K. A distinct feature in Fig. 3 is that a shoulder appears at the high-energy side of the phonon peak of the $A$ free exciton transition when the temperature is above $80 \mathrm{~K}$. Moreover, the relative intensity of this shoulder with respect to the LO phonon peak of the FXA increases with temperature so that it develops into a well-resolved peak when the temperature is above $200 \mathrm{~K}$, as indicated by the dotted line in Fig. 4. At room temperature, the energy separation between this new peak and the phonon peak of FXA is about $25.4 \mathrm{meV}$. It is also found that this energy separation basically remains unchanged with temperature. Because of the much smaller energy distance $(\sim 7 \mathrm{meV})$ between FXA and FXB, the LO

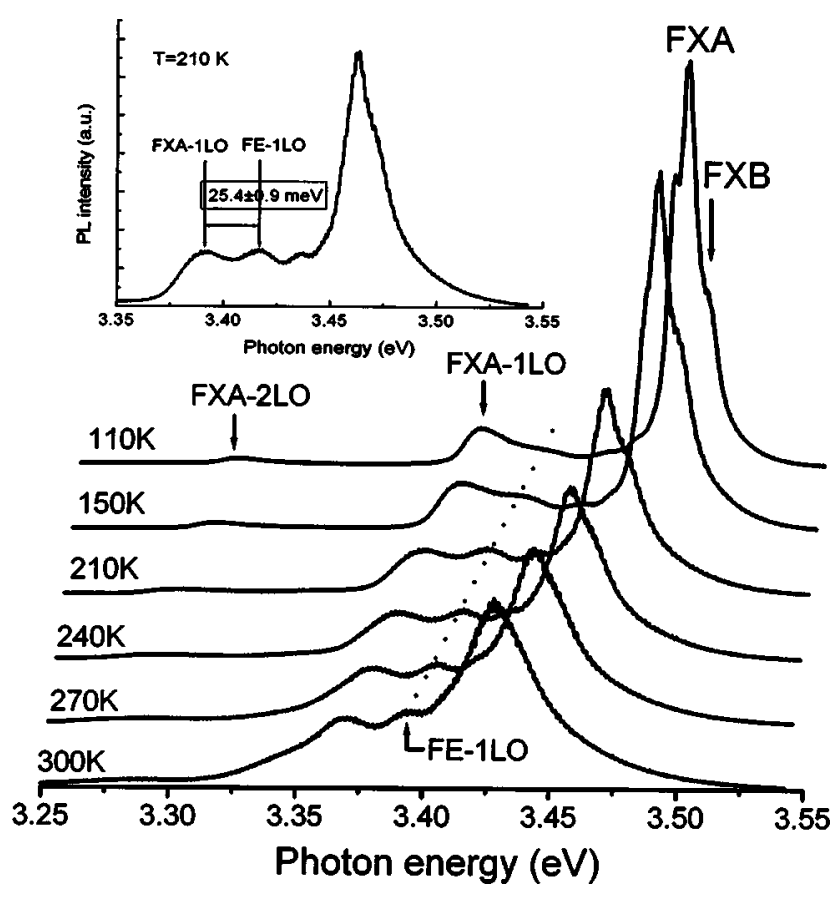

FIG. 4. Exciton resonant luminescence lines and their LO phonon peaks at higher temperatures. The curves are shifted in the $x$ direction for clarity. The inset shows a PL spectrum at $210 \mathrm{~K}$. 
phonon peak of FXB can be immediately ruled out as an origin of the new peak. It is not the phonon peak of the $2 S$ state of the $A$ free exciton because of the smaller energy separation $(\sim 18.7 \mathrm{meV})$ between $1 S$ and $2 S$ states and the very weak transition probability of the $2 S$ state., ${ }^{3,4,8}$ We attribute this peak to the LO phonon peak of the band-to-band transition, denoted by FE-1LO.

It is known that the thermal dissociation process of the free excitons is $F X A \rightarrow e+h{ }^{3}$ where $e$ and $h$ stand for free electron and free hole, respectively. At higher temperatures, the thermal dissociation rate of free excitons increases with temperature. As a result, the relative intensity of the band-toband transition increases with the temperature. Unfortunately, the band-to-band transition is difficult to be resolved due to significant thermal broadening (exponential dependence on temperature $)^{14,15}$ and thermal distribution of free excitons at high temperatures. However, for the phononassisted free exciton luminescence process, the transition probability depends linearly on the exciton kinetic energy and its linewidth increases only linearly with temperature according to theoretical model. ${ }^{16}$ Therefore, it is possible to resolve the phonon peaks of the free exciton transition and the band-to-band transition. Because the energy separation between the band-to-band transition and the free exciton transition is just the binding energy of free exciton, the binding energy can be directly determined from the energy spacing between the phonon peaks of the band-to-band and the free exciton transitions. Therefore, the binding energy of the $A$ free exciton should be $25.4 \pm 0.9 \mathrm{meV}$. The error is mainly from the man-made error in determination of the PL peaks. This value is slightly larger than $24.9 \mathrm{meV}$ indirectly determined from the energy separation between both transitions from the $1 S$ and $2 S$ state of free exciton at low temperature. This indicates that the hydrogenic model is still a good approximation to describe the energy states of the free excitons in heteroepitaxial GaN thin films.
In summary, the LO phonon-assisted PL spectra of GaN films grown on sapphire are investigated. The LO phonon peak of the band-to-band transition is clearly observed at higher temperatures. Combined with the phonon peak of the free exciton transition, this leads to a direct determination of the free exciton binding energy. It is found that the binding energy of free excitons is $25.4 \pm 0.9 \mathrm{meV}$.

The work is supported by an HKU Research Grant (No. 10203533). One of the authors (S.J.X.) thanks Z. Y. Xu for her English corrections of the manuscript.

${ }^{1}$ B. Monemar, J. Cryst. Growth 189, 1 (1998).

${ }^{2}$ M. Smith, G. D. Chen, J. Z. Li, J. Y. Lin, H. X. Jiang, A. Salvador, W. K. Kim, O. Aktas, A. Botchkarev, and H. Morkoc, Appl. Phys. Lett. 67, 3387 (1995).

${ }^{3}$ D. Volm, K. Oettinger, T. Streibl, D. Kovalev, M. Ben-Chorin, J. Diener, B. K. Meyer, J. Majewski, L. Eckey, A. Hoffmann, H. Amano, I. Akasaki, K. Hiramatsu, and T. Detchprohm, Phys. Rev. B 53, 16543 (1996).

${ }^{4}$ W. Shan, B. D. Little, A. J. Fischer, J. J. Song, B. Goldenberg, W. G. Perry, M. D. Bremser, and R. F. Davis, Phys. Rev. B 54, 16369 (1996).

${ }^{5}$ D. C. Reynolds, D. C. Look, W. Kim, A. Ösgür, A. Botchkarev, A. Salvador, H. Morkoç, and D. N. Talwar, J. Appl. Phys. 80, 594 (1996).

${ }^{6}$ M. Steube, K. Reimann, D. Fröhlich, and S. J. Clarke, Appl. Phys. Lett. 71, 948 (1997).

${ }^{7}$ A. A. Yamaguchi, Y. Mochizuki, H. Sunakawa, and A. Usui, J. Appl. Phys. 83, 4542 (1998).

${ }^{8}$ B. J. Skromme, J. Jayapalan, R. P. Vaudo, and V. M. Phanse, Appl. Phys. Lett. 74, 2358 (1999).

${ }^{9}$ S. J. Xu, W. Liu, and M. F. Li, Appl. Phys. Lett. 77, 3376 (2000).

${ }^{10}$ B. K. Meyer, A. Hoffmann, and P. Thurian, in Group III Nitride Semiconductor Compounds: Physics and Applications, edited by B. Gil, (Oxford University Press, London, 1998), Chap. 7.

${ }^{11}$ R. J. Elliott, Phys. Rev. 108, 1384 (1957).

${ }^{12}$ C. F. Klingshirn, Semiconductor Optics (Springer, Berlin, 1995), p. 243.

${ }^{13}$ J. Jayapalan, B. J. Skromme, R. P. Vaudo, and V. M. Phanse, Appl. Phys. Lett. 73, 1188 (1998).

${ }^{14}$ I. A. Buyanova, J. P. Bergman, B. Monemar, H. Amano, and I. Akasaki, Mater. Sci. Eng., B 50, 130 (1997).

${ }^{15}$ W. Liu, M. F. Li, S. J. Xu, K. Uchida, and K. Matsumoto, Semicond. Sci. Technol. 13, 769 (1998).

${ }^{16}$ S. Permogorov, in Excitons, edited by E. I. Rashba and M. D. Sturge (North-Holland, Amsterdam, 1982), p. 177. 
Applied Physics Letters is copyrighted by the American Institute of Physics (AIP). Redistribution of journal material is subject to the AIP online journal license and/or AIP copyright. For more information, see http:/ojps.aip.org/aplo/aplcr.jsp

Copyright of Applied Physics Letters is the property of American Institute of Physics and its content may not be copied or emailed to multiple sites or posted to a listserv without the copyright holder's express written permission. However, users may print, download, or email articles for individual use. 